\title{
Decaying Swirl Flow Impact on Developing Laminar Pipe Flow
}

\author{
Mohammad O. Hamdan \\ American University of Sharjah \\ M-109C, University City, Sharjah, United Arab Emirates \\ mhamdan@aus.edu
}

\begin{abstract}
The impact of decaying swirl flow on developing laminar flow is investigated numerically in this article. The effect of swirl flow is investigated under constant wall temperature. The impact of the decaying swirl is described using swirl number, friction coefficient and Nusselt number. The results show that decaying swirl enhances local heat transfer by $40 \%$ but that comes at $300 \%$ increase in local friction factor when compared to the developing flow with no swirl. Hence the insertion of swirl generator in tube heat exchanger reduces the size of the heat exchanger but it increases pumping system size and cost. Also, the results show that pressure does not always decrease in the flow direction since it could increase and then decreased depending on the swirl strength.
\end{abstract}

Keywords: Decaying Swirl, Heat Augmentation, Laminar Developing Flow.

\section{Introduction}

The demand of improved heat removal increases with every engineering application. The need for more compact improved electronic systems has drove the increase of heat generation per device and per device volume. The heat removal of fully developed flow is not enough to meet the demands to heat removals. Hence developing flow is being used in many applications with other techniques such as improving fluid thermal properties by adding nanotubes additives [1], increasing local heat transfer coefficient through jet impingements [2-6], and improving wall contact with the fluid using porous inserts [7-11].

The heat transfer in fully developed laminar flow is limited at $N u=3.66$ for pipe with constant wall temperature [12]. One approach to enhance the heat transfer in laminar flow is by avoiding the fully developed region and keeping the flow in the developing region using short flow path. Swirl flow is an effective way to enhance heat transfer in the laminar developing region [13].

Swirl flows are widely used in different engineering applications such as chemical and mechanical mixing and separation devices, combustion chambers, turbo machinery, fusion reactors, pollution control devices [14] and flow measurements. Based on the swirl generator techniques, the swirl flows can be classified into two types which are uniform continuous swirl and none-uniform decaying swirl. The uniform continuous swirl flow is generated by spiral axial path, hence it sustains its swirl behaviour along the axial direction of the flow path. While the none-uniform decaying swirl is generated at the inlet and its swirl behaviour decays by viscous dissipation along the axial direction of the flow [15].

The swirl flow in the entrance region of a laminar flow has been explored by Kiya et al. [16] who have reported that the entrance length dramatically extended when swirl flow is introduced. The validity of numerical analysis of a decaying swirl in laminar flow in annulus channel has been reported in literature [17] where the flow fields have been explored versus an analytical solution. Ayinde [18] reported that the swirl flow exponentially decay after $x / D=16$ when fully developed laminar velocity profile is used at the pipe inlet.

The behaviour of swirl flow in turbulent flow has been investigated extensively in the literature [19-24]. The heat transfer in turbulent decaying swirl has been experimentally Chang and Dhir [19], who reported that heat transfer augmentation is due to high axial velocity near the wall and due to high turbulence level near the centre of the pipe. It has been shown in literature [20] that the rate of decaying swirl in turbulent pipe flow varies with the Reynolds number behave in similar way as the friction factor. In turbulent flow, a high heat augmentation of $98 \%$ enhancement has been reported by Yilmaz et al [21]. Using radial guided vane swirl generators, an experimental investigation [22] showed a 150\% augmentation in Nusselt number relative to that of the fully developed turbulent axial flow with a constant heat flux boundary condition. A propeller-type swirl generator has been investigated in turbulent flow [23] and showed a high 
improvement in Nusselt number with improvement ranging from $18.1 \%$ to $163 \%$. The effect of swirl generator length on heat transfer of turbulent has been explored by Eiamsa-ard et al. [24] who reported that shorter swirl generator produce less heat augmentation and less friction losses. The decaying swirl in turbulent flow has been investigated numerically by different research groups [25-27]. Parchen and Steenbergen [25] reported that the standard $k-\varepsilon$ model predicts the decay rate but it fails to show the sensitivity of initial velocity distribution on swirl decay. Numerical investigation [26] of the swirl turbulent flow has showed that the eddy-viscosity turbulence model fails to predict the combined vortex observed experimentally. Najafi et al. reported [27] that RSM turbulent model with twolayer zone is generally more powerful than others turbulence models in modelling decaying swirl flow.

The objective of this paper is to evaluate heat enhancement using swirl flow with uniform axial inlet velocity and compares the heat performance versus uniform entrance velocity with no swirl flow. The study reports the heat enhancement under constant wall temperature.

\section{Mathematical Modelling}

The uniform appearance will assist the reader to read paper of the proceedings. It is therefore suggested to authors to use the example of this file to construct their papers. This particular example uses an American letter format with 25 $\mathrm{mm}$ margins left, right, top and bottom.

All text paragraphs should be single spaced, with first line intended by $7 \mathrm{~mm}$. Double spacing should NOT be used anywhere in the manuscript. Position and style of headings and subheadings should follow this example. One empty line (11 pt) should be left between every two consecutive sections. Two empty lines should be left before and after the abstract.

A schematic diagram for the problem under consideration is shown in Fig. 1. The flow is three dimensional since it represents a developing flow that moves axially in the $x$-direction while rotating around the $x$-axis. Fig. 1 presents a two dimensional domain while the third direction is counted using the axisymmetric swirl around $x$-axis. The fluid inters at uniform velocity $\left(U_{i}\right)$ and under forced vortex $(\omega)$ around $x$-axis with a uniform temperature $\left(T_{i}\right)$. The flow is assumed laminar, single phase, with no viscous dissipation. The fluid is assumed homogenous and incompressible with constant properties. No heat is produced from viscous dissipation. The numerical analysis is carried out using finite volume approach via commercial software ANSYS-FLUENT.

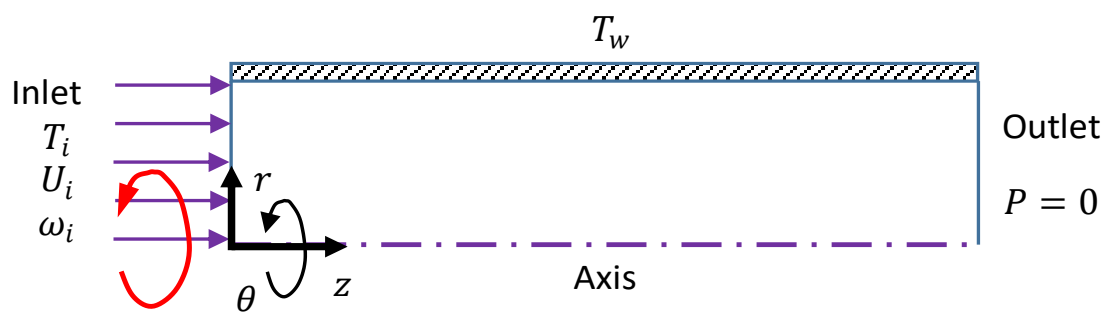

Fig. 1: A schematic diagram showing the problem under consideration with boundary conditions.

With reference to the previous assumption statements, the continuity, the Navier-Stokes and energy equations are enforced and are written in index notation form as follow:

$$
\begin{gathered}
\frac{\partial u_{i}}{\partial x_{i}}=0 \\
\rho u_{j} \frac{\partial u_{i}}{\partial x_{j}}=-\frac{\partial P}{\partial x_{i}}+\frac{\partial}{\partial x_{j}}\left[\mu\left(\frac{\partial u_{i}}{\partial j}+\frac{\partial u_{j}}{\partial x_{i}}\right)\right] \\
\rho u_{j} \frac{\partial u_{i}}{\partial x_{j}}=-\frac{\partial P}{\partial x_{i}}+\frac{\partial}{\partial x_{j}}\left[\mu\left(\frac{\partial u_{i}}{\partial j}+\frac{\partial u_{j}}{\partial x_{i}}\right)\right]
\end{gathered}
$$


The effect of swirl is reported as function of swirl number, $S$. The swirl number $(S)$ is a measure of swirl intensity which is defined as the ratio of the axial flux of angular momentum to the axial flux of axial momentum which is expressed expressed as follow [15]:

$$
S=\frac{\int_{0}^{a} \rho \omega u r^{3} d r}{R \int_{0}^{a} \rho u^{2} r d r}
$$
follow:

The inlet swirl number for forced vortex $(\omega=$ constant $)$ and incompressible flow inside a pipe can be defined as

$$
S_{i}=\frac{\omega_{i} a}{2 u_{i}}
$$

The effect of heat transfer is demonstrated by calculating Nusselt number at the wall. The Nusselt number is calculated as follow:

$$
N u=\frac{h D}{k}=\frac{q /\left(T_{w}-T_{b}\right) D}{k}
$$

The pressure loss is considered an important factor to study when comparing heat transfer effect of different mechanism such as swirl flow. The optimum method to implement for heat transfer is the one that require the smallest pumping power (i.e. produce the least pressure drop). The pressure loss is evaluated by calculating Darcy friction factor using equation (7).

$$
f=\frac{8 \tau_{w}}{\rho u_{i}^{2}}
$$

\section{Numerical Analysis}

A uniform fine quad map mesh is used with minimum cell size set equal to 0.02 of the pipe diameter. The numerical convergence has been assumed once the relative errors reach $10^{-9}$ for all the parameters. A grid refinement procedure has been performed through numerical experimentations to assure the grid independence. As shown in Fig. 2, a grid independence study is carried out by testing three different grid sizes and monitoring the dimensionless axial velocity at specific $x$-location in the developing region. The velocity profile is produce at $X=1$ for $\operatorname{Re}=100$ and $S_{i}=2$.

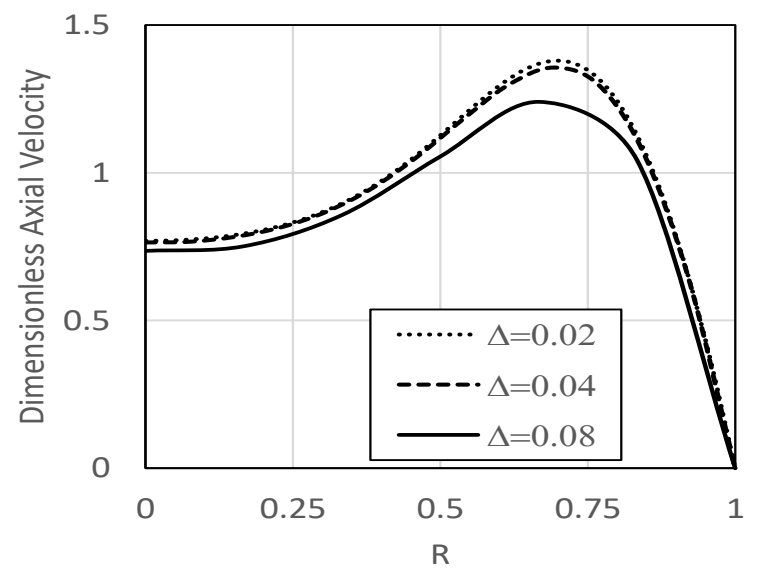

Fig. 2: The dimensionless axial velocity profile at $x=1$ for three different mesh sizes with $R e=100$ and $S_{i}=2$. 
To assure the validity of the numerical solution, the velocity profile, for the fully developed case with zero swirl $\left(S_{i}=0\right)$, is compared with the analytical solution $\left[u / u_{i}=2\left(1-(r / a)^{2}\right)\right]$ as shown in Fig. 3.

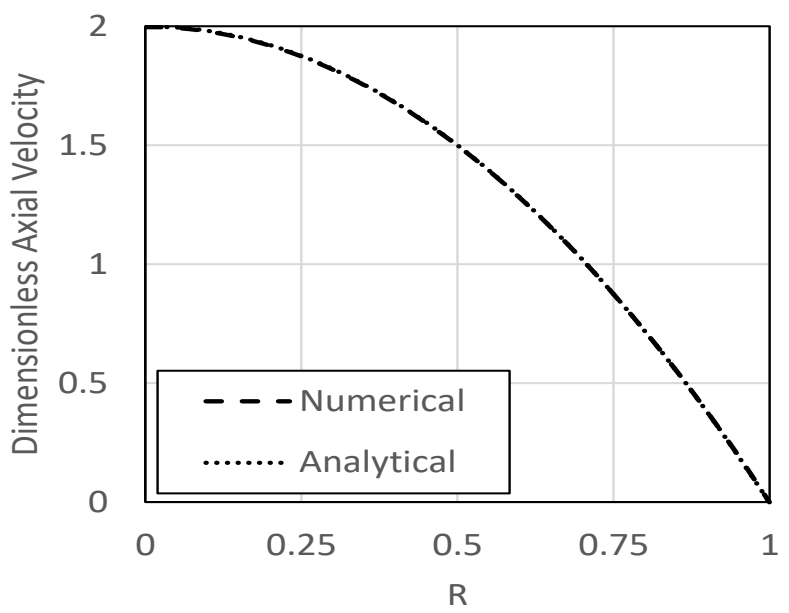

Fig. 3: Fully developed dimensionless axial velocity compared to analytical solution for $R e=100$ and $S_{i}=0$.

\section{Results and Discussion}

The results are calculated for different angular Reynolds numbers $(R e)$ and inlet swirl numbers $\left(S_{i}\right)$. The effect of inlet swirl number $\left(S_{i}\right)$ on the dimensionless axial velocity $(U)$ at distance $X=1$ is shown in Fig. $4 \mathrm{a}$. The swirl flow pushes the fluid toward the wall of the pipe due to the centrifugal force causing more fluid to flow near the wall as observed in Fig. 4. At the same location $(X=1)$, as Reynolds number increases, the boundary layer thickness decreases allowing higher axial velocity near the pipe wall as shown in Fig. $4 \mathrm{~b}$.

The dimensionless tangential velocity is plotted versus dimensionless radial coordinate in Fig. 5a for $R e=100$ and Fig. $5 \mathrm{~b}$ for $S_{i}=2$. Since dimensionless inlet tangential velocity $(W)$ is related to swirl number as $S_{i}=\omega_{i} a / 2 u_{i}=$ $W_{i} / s$, it is expected that the dimensionless tangential velocity $(W)$ increases as swirl number increases. For a fixed swirl number $\left(S_{i}=2\right)$, Fig. 5b shows that as the dimensionless axial inlet velocity increases (represented by Reynolds number), the dimensionless swirl velocity increases and the peak value shifts toward the pipe. As explained earlier in Fig. 4b, as Reynolds number increases, the boundary layer thickness decreases allowing higher tangential velocity near the pipe wall.
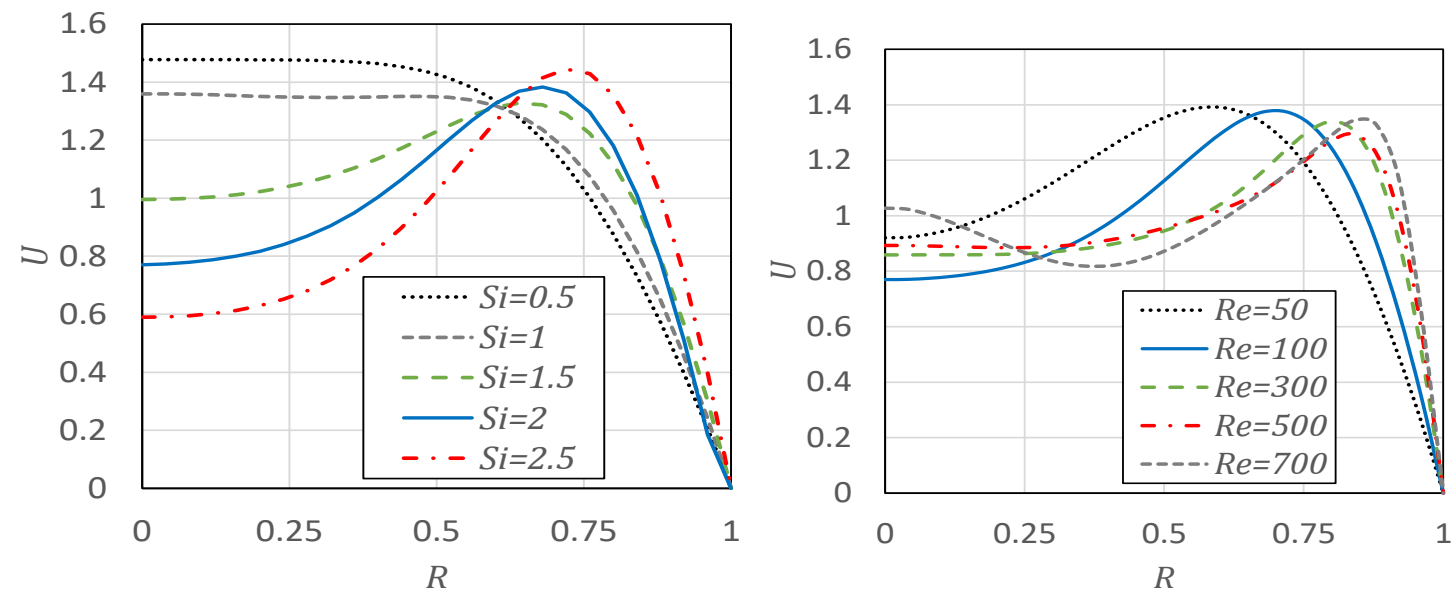

Fig. 4: The dimensionless axial velocity profile with respect to dimensionless radial coordinate at $X=1$ for (a) $R e=100$ with different $S_{i}$ values, and (b) $S_{i}=2$ with different $R e$ values. 

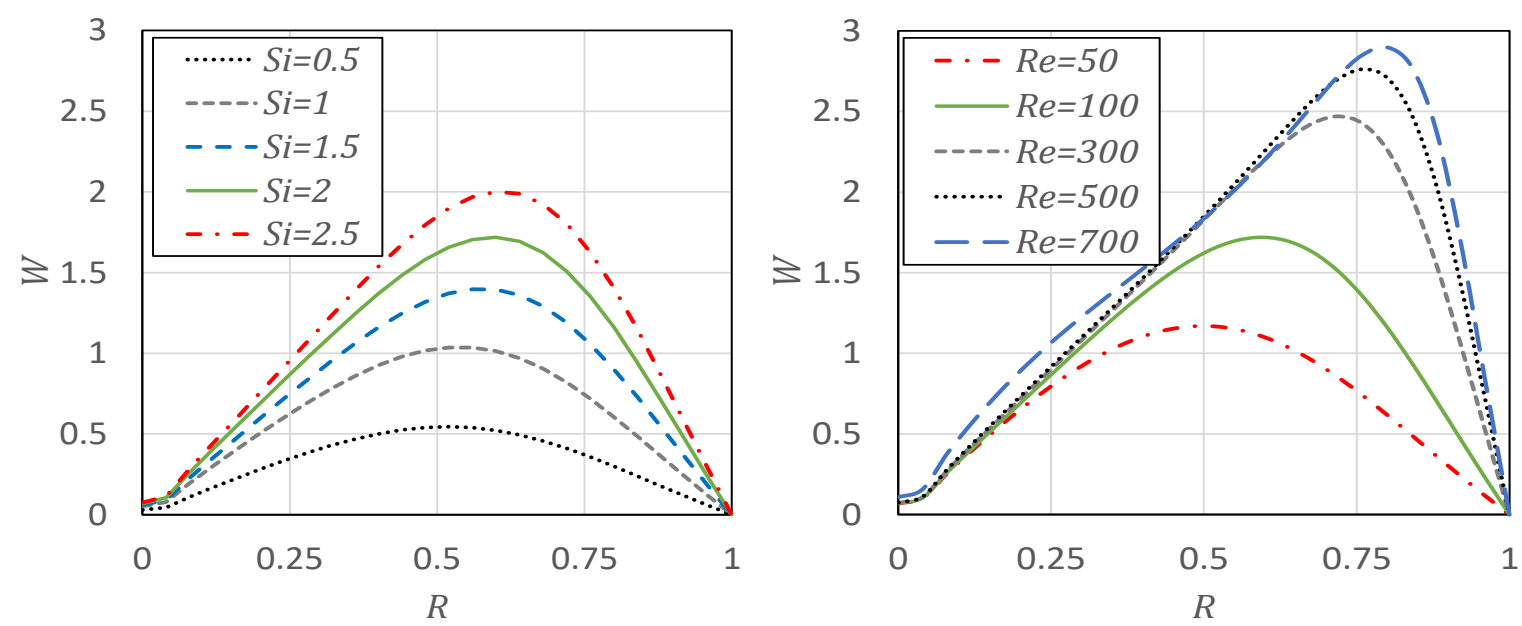

Fig. 5: The dimensionless swirl velocity profile with respect to dimensionless radial coordinate at $X=1$ for (a) $R e=100$ with different $S_{i}$ values, and (b) $S_{i}=2$ with different $R e$ values.

The effect of centrifugal force is observed on pressure as shown in Fig. 6a. It is clear that as angular velocity increases, the dimensionless pressure near the wall increases while decreases near the pipe centre. The swirl flow affects how pressure behaves at the centreline. The static pressure at the centreline is under two conflicting behaviours, the first one that pressure will decrease as flow moves in the $\mathrm{x}$-direction due to friction losses, while the second effect is that pressure increases as swirl decays and centrifugal force deteriorates. As the decaying swirl weakens with axial direction, the pressure at the eye of the swirl starts to build-up. This behaviour is similar to diffuser followed with a nozzle in which pressure will increase in the diffuser section and then will decrease in the nozzle section. Hence overall centreline pressure increases at the entrance of the pipe, then starts to decrease after reaching a peak value as shown in Fig. 6b. Fig. $6 \mathrm{~b}$ shows how the dimensionless pressure changes with respect to the dimensionless axial direction.
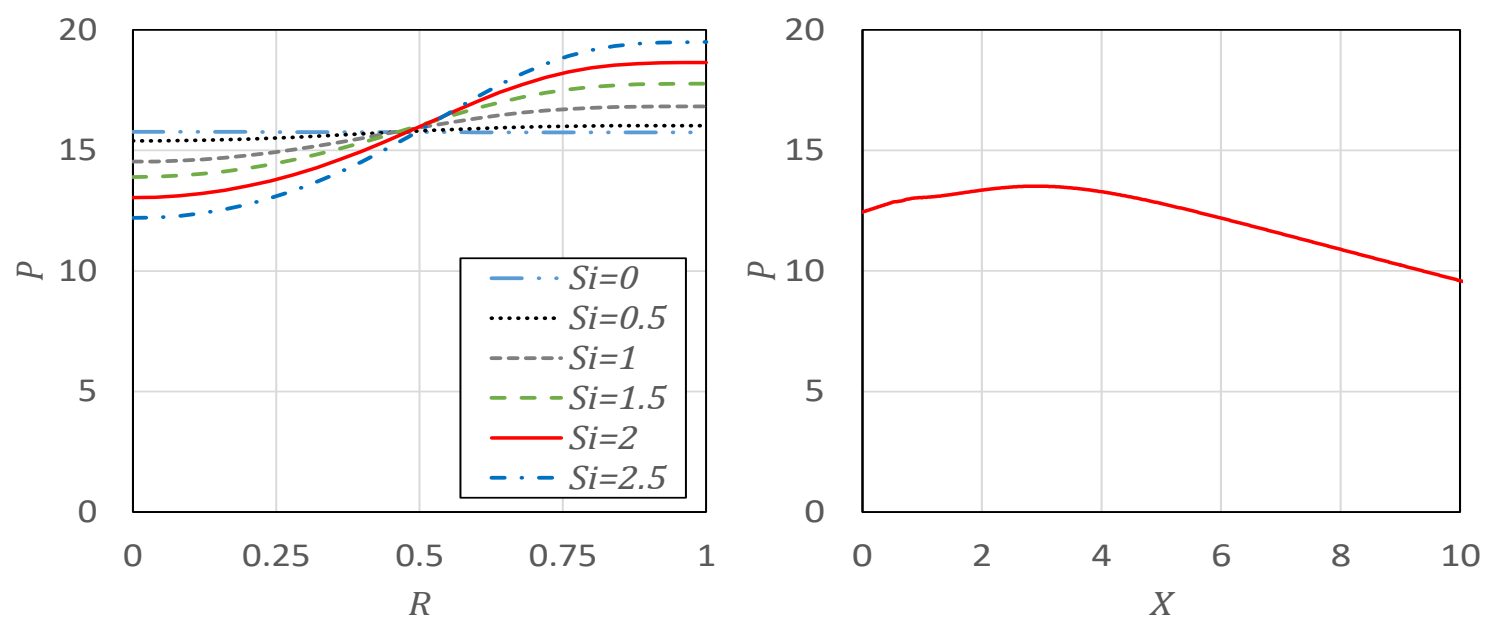

Fig. 6: (a) The dimensionless pressure versus the dimensionless radial direction at $X=1$ for $R e=100$ and different $S_{i}$ values, (b) the dimensionless centreline pressure versus the dimensionless axial direction for $R e=100$ and $S_{i}=2$.

The local swirl number variation with axial coordinate is shown in Fig. 7. The swirl number has maximum value at the inlet as shown in Fig. 7. The swirl number decays in the axial direction due to viscosity friction forces. The rate of swirl decay with respect to $\mathrm{x}$-direction has maximum value at the inlet and keeps decreasing as fluid moves in the axial direction. The reduction in the rate of swirl decay is due to friction losses which has maximum value near the wall. The high friction shear at the entrance is due the high rate of deformation in of the fluid at that point which starts to decrease as 
boundary layer starts to form. Fig. 7b shows the variation of swirl number with the axial coordinate for different values of Reynolds number. A high Reynolds number means that more angular momentum is transported with the flow stream which extends the length of swirl flow. It is very clear in Fig. 7b that rate of decay in swirl number increases as Reynolds number decreases high Reynolds number means more momentum flux is moving toward the axial direction which strengthen the swirl flow.

The expense of adding swirl generator on pumping pressure is shown in Fig. 8a which shows how local Darcy friction factor changes with respect to $x$-direction for different swirl number. The friction factor approaches the known Darcy friction factor $(f=64 / R e)$ of a laminar fully developed flow. In Fig. 8a, the value of friction factor for fully developed flow is $f=0.64$ when $R e=100$. As shown in Fig. 8a that the friction factor increases with the increase in inlet swirl number. The increase in swirl number causes an increase of fluid flow near the wall causing higher velocity gradient causing higher wall shear stress, hence a higher friction factor. Also, higher swirl number means higher velocity near the wall which will cause higher shear force at the wall. It is clear that swirl flow increases the entrance region length since it pushes on the boundary layer causing thinner boundary layer to form at the same $\mathrm{x}$-location.

The effect of decaying swirl on constant wall temperature case is shown in Fig. 8b. As shown in Fig. 8b, the Nusselt for the fully developed flow matches literature [12] values of 3.66. As expected, Nusselt number decreases as fluid moves in the x-direction since boundary layer starts to increase causing a reduction in heat transfer. The increase in swirl flow causes an increase in heat transfer as observed in the figure.
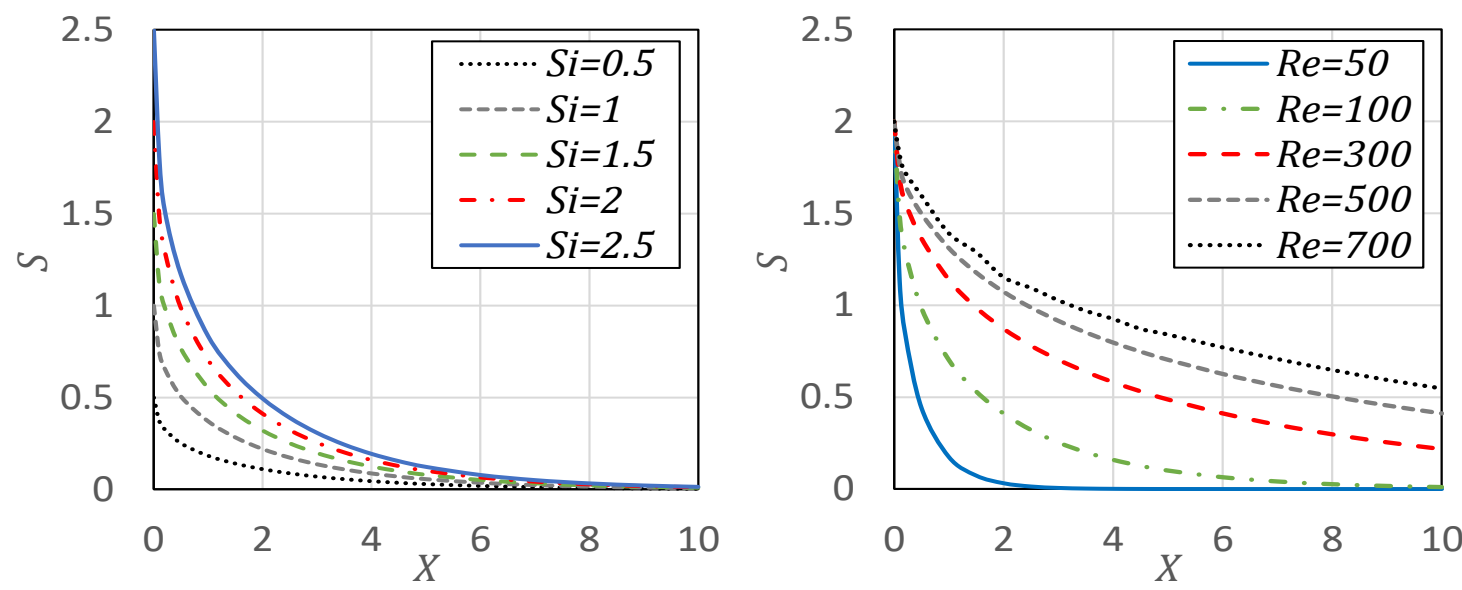

Fig. 7: The variation of swirl number with respect to dimensionless x-direction for (a) $R e=100$ and different $S_{i}$ values, and (b) $S_{i}=2$ and different $R e$ values.
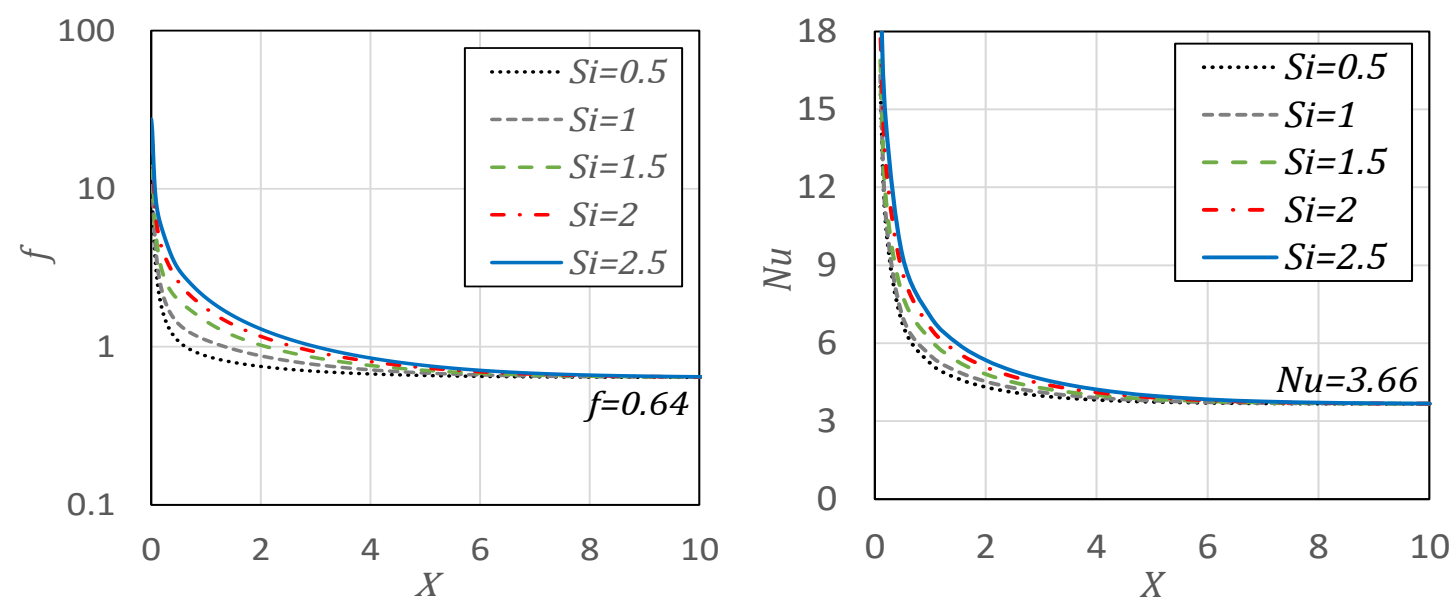

Fig. 8: The effect of swirl number for $R e=100$ with constant wall temperature on (a) local wall friction factor and (b) local Nusselt number. 


\section{Conclusions}

Introducing swirl flow at the entrance of a pipe with laminar flow promotes heat transfer. The swirl flow provide higher heat transfer compared to developing flow, hence it will have even higher heat transfer when compared to fully developed flow. The enhancement in heat transfer is associated to an increase in pressure losses. The swirl flow increases the length of the entrance region which means higher average heat transfer coefficient. The variation of centreline pressure is non-linear and it depends on inlet swirl and Reynolds numbers.

\section{References}

[1] E. Elnajjar, Y. Haik, M. O. Hamdan, and S. Khashan, "Heat transfer characteristics of multi-walled carbon nanotubes suspension in a developing channel flow," Heat and Mass Transfer, vol. 49, pp. 1681-1687, 2013.

[2] M. O. Hamdan, E. Elnajjar, and Y. Haik, "Measurement and modeling of confined jet discharged tangentially on a concave semicylindrical hot surface," Journal of Heat Transfer, vol. 133, p. 122203, 2011.

[3] M. Hamdan and M. Al-Nimr, "Thermal augmentation in internal cooling passage by converting impingement jet to induced swirl flow," in Proceedings of 6th International Conference on Computational Heat and Mass Transfer, pp. 18-21, 2009.

[4] E. Elnajjar, M. O. Hamdan, and Y. Haik, "Experimental Investigation of Internal Channel Cooling Via Jet Impingement," FDMP: Fluid Dynamics \& Materials Processing, vol. 9, pp. 77-89, 2013.

[5] M. O. Hamdan and A. Y. Hayek, "Effect of Cavity Size on Confined Slot Jet Impingement Cooling," in ASME 2013 Heat Transfer Summer Conference collocated with the ASME 2013 7th International Conference on Energy Sustainability and the ASME 2013 11th International Conference on Fuel Cell Science, Engineering and Technology, 2013, pp. V002T04A013-V002T04A013.

[6] E. Elnajjar, M. Hamdan, and Y. Haik, "Experimental Investigation of Impinging Jet Flow on a Heated Curved Surface," in 6th International Conference on Thermal Engineering, Istanbul, Turkey, 2012.

[7] M. Alkam, M. Al-Nimr, and M. Hamdan, "Enhancing heat transfer in parallel-plate channels by using porous inserts," International Journal of Heat and Mass Transfer, vol. 44, pp. 931-938, 2001.

[8] M. Alkam, M. Al-Nimr, and M. Hamdan, "On forced convection in channels partially filled with porous substrates," Heat and Mass Transfer, vol. 38, pp. 337-342, 2002.

[9] M. Hamdan, M. Al-Nimr, and M. Alkam, "Enhancing forced convection by inserting porous substrate in the core of a parallel-plate channel," International Journal of Numerical Methods for Heat \& Fluid Flow, vol. 10, pp. 502-518, 2000.

[10] M. Hamdan and A.-N. Moh'd A, "The use of porous fins for heat transfer augmentation in parallel-plate channels," Transport in porous media, vol. 84, pp. 409-420, 2010.

[11] M. O. Hamdan, "An empirical correlation for isothermal parallel plate channel completely filled with porous media," Thermal Science, vol. 17, pp. 1061-1070, 2013.

[12] Y. Cøengel, "Heat and mass transfer: a practical approach," McGraw-Hill, New York, 2007.

[13] M. Hamdan, "Numerical analysis of Enhanced Heat Transfer in Developing Laminar Pipe Flow using Decaying Swirl at the Inlet," Journal of Enhanced Heat Transfer.

[14] S. Liu and M. Sakr, "A comprehensive review on passive heat transfer enhancements in pipe exchangers," Renewable and Sustainable Energy Reviews, vol. 19, pp. 64-81, 2013.

[15] A. K. Gupta, D. G. Lilley, and N. Syred, "Swirl flows," Tunbridge Wells, Kent, England, Abacus Press, 1984,488 p., 1984.

[16] M. Kiya, S. Fukusako, M. Arie, "Laminar swirling flow in the entrance region of a circular pipe," JSME, vol. 14, pp. 659-670, 1971.

[17] A. M. Jawarneh, G. H. Vatistas, and A. Ababneh, "Analytical approximate solution for decaying laminar swirling flows within a narrow annulus," Jordan Journal of Mechanical and Industrial Engineering (JJMIE), vol. 2, pp. 101109, 2008.

[18] T. Ayinde, "A generalized relationship for swirl decay in laminar pipe flow," Sadhana, vol. 35, pp. 129-137, 2010.

[19] F. Chang and V. Dhir, "Mechanisms of heat transfer enhancement and slow decay of swirl in tubes using tangential injection," International Journal of Heat and Fluid Flow, vol. 16, pp. 78-87, 1995. 
[20] W. Steenbergen and J. Voskamp, "The rate of decay of swirl in turbulent pipe flow," Flow measurement and instrumentation, vol. 9, pp. 67-78, 1998.

[21] M. Yilmaz, Ö. Çomakli, and S. Yapici, "Enhancement of heat transfer by turbulent decaying swirl flow," Energy conversion and management, vol. 40, pp. 1365-1376, 1999.

[22] M. Yilmaz, O. Comakli, S. Yapici, and O. N. Sara, "Heat transfer and friction characteristics in decaying swirl flow generated by different radial guide vane swirl generators," Energy Conversion and Management, vol. 44, pp. 283300, 2003.

[23] B. A. Saraç and T. Bali, "An experimental study on heat transfer and pressure drop characteristics of decaying swirl flow through a circular pipe with a vortex generator," Experimental Thermal and Fluid Science, vol. 32, pp. 158$165,2007$.

[24] S. Eiamsa-Ard, C. Thianpong, P. Eiamsa-Ard, and P. Promvonge, "Convective heat transfer in a circular tube with short-length twisted tape insert," International communications in heat and mass transfer, vol. 36, pp. 365-371, 2009.

[25] W. Steenbergen, "An Experimental and Numerical Study of Turbulent Swirling Pipe Flows," 1998.

[26] A. Hoekstra, J. Derksen, and H. Van Den Akker, "An experimental and numerical study of turbulent swirling flow in gas cyclones," Chemical Engineering Science, vol. 54, pp. 2055-2065, 1999.

[27] A. Najafi, M. Saidi, M. Sadeghipour, and M. Souhar, "Numerical analysis of turbulent swirling decay pipe flow," International communications in heat and mass transfer, vol. 32, pp. 627-638, 2005. 